\title{
Procesos de comunicación en la clase de matemáticas de grado $5^{\circ 1}$
}

\section{Communication processes in the class of 5th grade math}

Processos de comunicação na classe de matemática $5^{\circ}$ ano

Recibido: mayo de 2013

Aceptado: agosto de 2013
Liceth Katherine Beltrán Perdomo ${ }^{2}$

Andrés Felipe Caro ${ }^{3}$

Diana Paola Fernández Herrán ${ }^{4}$

Lina Estefanía Rozo Castañeda ${ }^{5}$

\section{Resumen}

En este artículo se muestran los resultados de un proyecto de campo realizado mediante la utilización de una metodología de estudio de caso, centrado en las estrategias utilizadas en los procesos de comunicación frente a la relación estudiante-docente de la clase de matemáticas en grado quinto, a partir de la aplicación de unos instrumentos de investigación diseñados y basándolo en lo propuesto por Camacaro (2008), Chevallard (1991), Cross (2000), Kaplun (1998), Rojas (2009) y otros, se logró evidenciar la falta de afinidad entre las opiniones de lo percibido por el docente y por el estudiante y las concepciones de superioridad que se tiene de los roles que están en juego en el aula.

Palabras clave: Comunicación; estrategias; profesor; estudiante; clase de matemáticas; relaciones interpersonales; análisis y reflexión sobre la enseñanza.

\section{Abstract}

This article presents the results of a field project conducted by using a case study methodology, focusing on the strategies used in communication processes against the student-teacher math class in fifth grade, from the application of investigation tools designed and proposed by basing it on Camacaro (2008), Chevallard (1991), Cross (2000), Kaplun (1998), Rojas (2009) and others, are able to demonstrate the lack of affinity between the views of what is perceived by the teacher and the student and perceptions of superiority we have of the roles that are at play in the classroom.

Keywords: Communication, strategies, teacher, student, math class, interpersonal relationships, analysis and reflection on teaching.

1 Articulo de Investigación.

2 Universidad Distrital Francisco José de Caldas, Bogotá, Colombia. Contacto: lizbek320@hotmail.com

3 Universidad Distrital Francisco José de Caldas, Bogotá, Colombia. Contacto: candres44@hotmail.com

4 Universidad Distrital Francisco José de Caldas, Bogotá, Colombia. Contacto: dianapaofh@hotmail.com

5 Universidad Distrital Francisco José de Caldas, Bogotá, Colombia. Contacto: linis_3602@hotmail.com 


\section{Resumo}

Este artigo apresenta os resultados de um projeto de campo realizada através de uma metodologia de estudo de caso, com foco nas estratégias utilizadas nos processos de comunicação contra a aula de matemática aluno-professor na quinta série, a partir da aplicação de ferramentas de investigação concebido e proposto por baseando-se em Camacaro (2008), Chevallard (1991), Cruz (2000), Kaplun (1998), Rojas (2009) e outros, são capazes de demonstrar a falta de afinidade entre os pontos de vista do que é percebido pelo professor e do aluno e percepções de superioridade que temos dos papéis que estão em jogo na sala de aula.

Palavras-chave: Comunicação, estratégias, professor, estudante, classe da matemática, relacionamento interpessoal, análise e reflexão sobre o ensino.

\section{Introducción}

Este trabajo de investigación surge como una experiencia en la formación escolar y como $\mathrm{EPPM}^{6}$ de cada uno de los integrantes del grupo de trabajo de la materia ambientes y mediaciones en el aula del grupo II del proyecto curricular LEBEM, en la Universidad Distrital Francisco José de Caldas, siendo en este espacio de formación donde se inicia un interés por las interacciones entre los individuos y el entorno social.

Por ello en este espacio, es posible evidenciar que la enseñanza de las matemáticas implica un proceso riguroso tanto de las personas que aprenden y las que enseñan, en cuanto los procesos enseñanza-aprendizaje y a su vez la comunicación que se lleva a cabo en el aula, desde el rol como estudiantes, en muchas ocasiones el miedo por las matemáticas o el gusto que haya por esta ciencia hace que los estudiantes muestren interés por la misma.

Retomando lo expuesto anteriormente y considerando la comunicación como un proceso importante en el desarrollo de las clases de matemáticas, por tanto para indagar acerca de dicha temática, a continuación se da paso a la pregunta que orientara: ¿Cuáles son las estrategias utilizadas por los docentes en los procesos de comunicación en la clase de matemáticas en los grados quintos $\left(5^{a}\right)$ del colegio distrital OEA de la jornada mañana?

\section{Marco teórico}

La comunicación es el motor de muchos de los avances en la práctica docente, debido a su importancia el docente hace uso de ella por medio del lenguaje, tanto verbal como no verbal. Y es en el uso de la comunicación entre profesor-estudiante donde se miran, sus diferentes interacciones ya sean buenas o malas. Estas interacciones se definen según Cros (2000), como situaciones asimétricas, ya que estas pueden afectar de manera directa en el proceso de enseñanza y aprendizaje dentro y fuera del aula.

Es importante mencionar que algunos de los factores que afectan y conforman la comunicación en el ambiente escolar se encuentran en; el contexto, los autores, el contenido y la interacción que según Camacaro (2008) determinan la manera en la cual se genera la comunicación en el aula a partir de normas y objetivos. En este sentido debemos tener en cuenta que en la comunicación se pueden producir obstáculos sociales, intelectuales, psicológicos, académicos, entre otros por parte de los estudiantes si los docentes no hacen una clase amena, con objetivos claros o ignoran las necesidades que presentan los estudiantes; haciendo que el interés por parte de los mismo sea solo por buenas notas y no por el deseo de aprender y obtener nuevos conocimientos.

Por ello, se tiene en cuenta el fin didáctico que guía la interacción que se desarrolla en el aula, esta exige que el docente logre emplear estrategias que 
orienten el rumbo de la clase y le permitan alcanzar el propósito de la misma, en situaciones comunicativas de armonía y respeto, según Cros (2003), considera que una parte de la actividad didáctica basada en el discurso docente se centra, en la medida de las relaciones de poder y de distancia que determina las situaciones de la clase.

Por último es importante mencionar la relación que existe entre los roles que se presentan en el aula de clase y los procesos comunicativos expuestos anteriormente, con el área de las matemáticas, para ello, se a tenido en cuenta las contribuciones realizadas por Brendefur \& Frykholm (citado por Rojas, 2009), que proponen tres clases de comunicación en el área de matemáticas.

- Comunicación contributiva, los estudiantes exponen sus ideas y estrategias e intentan profundizar en su aprendizaje y comprensión matemático.

- Comunicación reflexiva, se realizan diferentes reflexiones sobre las ideas realizadas por los estudiantes y el profesor, de tal forma que se pueda dar un mejor proceso de aprendizaje en el área de matemáticas.

- Comunicación instructiva, el docente toma las ideas expuestas por los estudiantes y sus ideas, logrando relacionarlas entre sí, para llegar a una sola y darla a conocer a los estudiantes.

Finalmente se menciona que las diferentes formas comunicativas del profesor, son necesarias al momento de integrar el discurso matemático del aula con las contribuciones de los estudiantes dentro del proceso de enseñanza y aprendizaje.

\section{Metodología}

Para el presente proyecto de investigación se tendrá en cuenta la metodología estudio de caso, en donde según Eisenhardt (1989) citado en Martínez (2006), lo define como: “...una estrategia de investigación dirigida a comprender las dinámicas presentes en contextos singulares.... Permitiendo la recopilación de información necesaria para dar respuesta al problema a partir de entrevistas semi-estructuradas y encuestas (ver instrumentos de recolección) aplicadas respectivamente a 6 estudiantes y 5 profesores del grado $5^{\circ}$ del colegio OEA de la localidad de Kennedy con el fin de observar los distintos puntos de vista de la población implicada con respecto a la comunicación entre el profesor-estudiante y estudiante-profesor.

Tabla 1

\begin{tabular}{|c|c|c|c|}
\hline 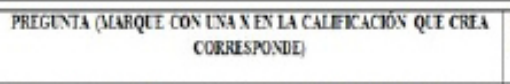 & 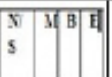 & PRECLXIis & BESPCISLLS \\
\hline \multirow{2}{*}{ 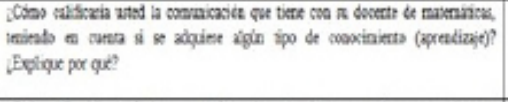 } & & 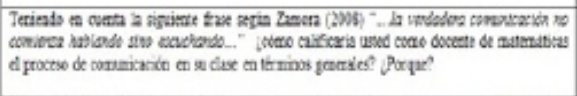 & \\
\hline & & \multirow{2}{*}{ 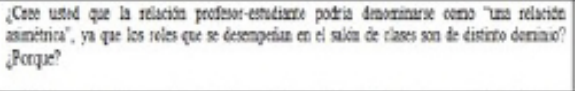 } & \\
\hline \multirow{2}{*}{ 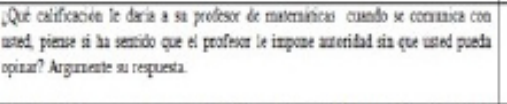 } & & & \\
\hline & & \multirow{2}{*}{ 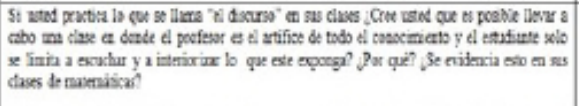 } & \\
\hline 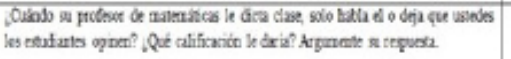 & & & \\
\hline 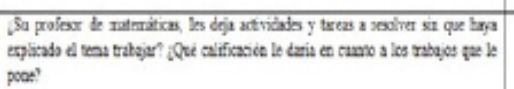 & & 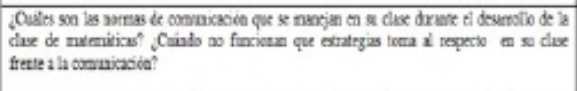 & \\
\hline \multirow{2}{*}{\multicolumn{2}{|c|}{ 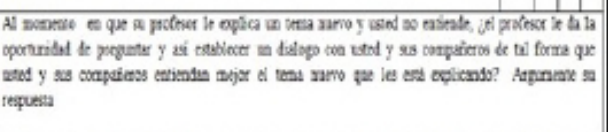 }} & 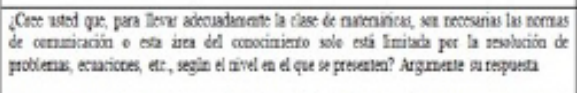 & \\
\hline & & 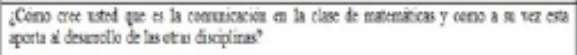 & \\
\hline \multicolumn{2}{|c|}{ 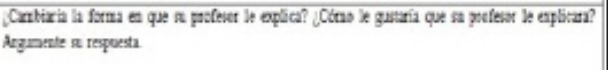 } & 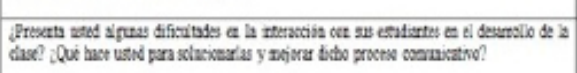 & \\
\hline \multicolumn{2}{|l|}{ Formato Encuestas /estudiantes } & \multicolumn{2}{|l|}{ Formato entrevistas /profesores } \\
\hline \multicolumn{4}{|c|}{ Instrumentos de recolección } \\
\hline
\end{tabular}

Fuente: Elaboración propia 
Finalmente para el análisis de resultados de las entrevistas y encuestas, se hará un análisis de tipo cualitativo según las respuestas de los docentes y de tipo cuantitativo según los estudiantes cuyos resultados clasificando la información correspondiente (ver instrumentos de análisis) a partir de las reflexiones hechas por los autores en cuanto a los procesos de comunicación y la relaciones profesor-estudiante partiendo de las bases teóricas expuestas principalmente por Kaplun (1998), Greimás (1994) y Camacaro (2008).

\section{Análisis de resultados}

Los resultados que se presentan a continuación son referente a las preguntas diseñadas en las entrevistas y la encuesta teniendo en cuanta la siguiente población:

Tabla 2

\begin{tabular}{|c|c|}
\hline $\begin{array}{l}\text { Estudiantes } \\
\text { Referente a la pregunta ¿Cuándo su profesor de } \\
\text { matemáticas le dicta clase, solo habla él...? }\end{array}$ & $\begin{array}{l}\text { Profesores } \\
\text {...Usted practica lo que se llama "el discurso", } \\
\text { en sus clases... }\end{array}$ \\
\hline $\begin{array}{l}\text { El } 50 \% \text { de los estudiantes respondieron que al } \\
\text { docente les deban la calificación de mala; ya } \\
\text { que no los dejaba opinar ni debatir entre ellos } \\
\text { mismos, solo importaba su opinión (docente). }\end{array}$ & $\begin{array}{l}\text {-Creo que si puede ser posible y creo que hoy } \\
\text { en dia existen aun muchos profesores que } \\
\text { dirigen sus clases de estas formas. } \\
\text {-En mi clase en especifico, yo no considero } \\
\text { que sea el unico capaz de expresar ideas que } \\
\text { enriquezcan de conocimientos a los demás. }\end{array}$ \\
\hline
\end{tabular}

Fuente: Elaboración propia

\section{Grafica 1}

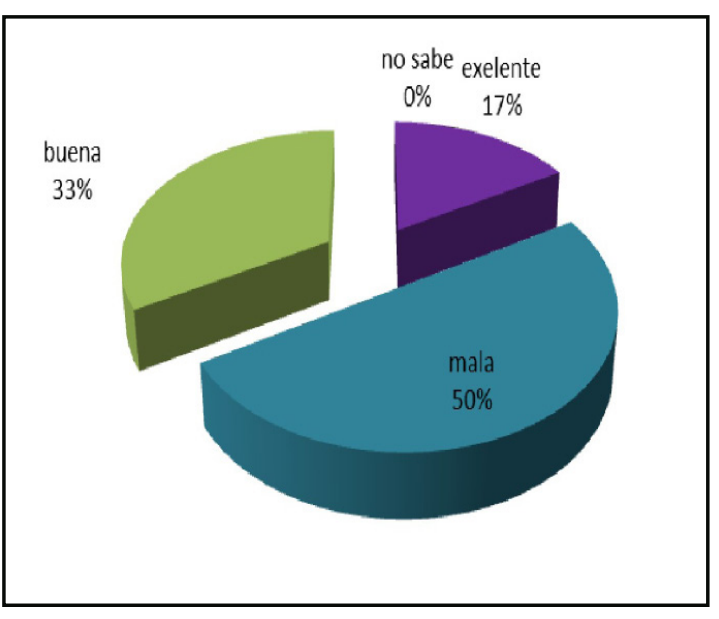

Fuente: Elaboración propia
Tabla 3

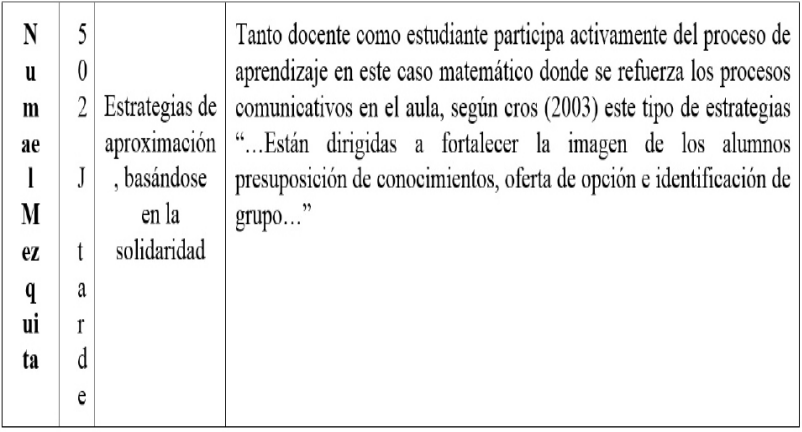

Fuente: Elaboración propia

Ahora, en cuanto a lo inferido en el caso de los profesores se puede decir que estos en un primer momento ignoran las falencias existentes en los procesos de comunicación, argumentando que a los estudiantes se les da oportunidades de participación e interacción, permitiendo generar la triangulación entre saber, profesor y estudiante que se busca plantear en el aula, aunque posteriormente, en algunos casos, se evidencia, la falta o necesidad de mejorar la comunicación que se debe buscar establecer entre estudiante-profesor, para lograr establecer buenas comunicaciones.

\section{Conclusiones}

Con la investigación realizada se pudo identificar las estrategias utilizadas en los procesos de comunicación frente a la relación estudiante-docente de la clase de matemáticas por parte de los docentes logrando hacer una relación entre la práctica y la teoría comparando la información recolectada con su respectivo análisis concluyendo en primer lugar que la comunicación en el aula de matemáticas en algunos de los casos se encontro regida por modelos pedagógico tradicionalistas, donde se identificaba al profesor como el artífice de todo conocimiento, ya que principalmente se encargaba de exponer el tema, ejemplificar, ejercitar y evaluar de forma sumativa, careciendo de una metodología de comunicación, ignorando las necesidades e intereses de los estudiantes. Por otro lado con respecto a la relación directa profesor-estudiante, se pudo evidenciar que las opiniones se encuentran muy fragmentadas, ya que mientras los profesores piensan que su 
metodología de trabajo es buena y consideran que a los estudiantes les agrada porque no opinan respecto a la misma, los estudiantes no se encuentran satisfechos con la comunicación que existe entre ellos, limitándose a un discurso explicativo.

Centrándonos en las estrategias de comunicación en el aula en las interacciones del docente-estudiante se logró evidenciar que los docentes en muchas ocasiones no usan adecuadamente los procesos de comunicación con los estudiantes; generando así un desinterés por parte de los mismos que de acuerdo a los tipos de comunicación expuestos por Brendefur y Frykholm (cit. Por Rojas, 2009) esta puede darse según las personas que se involucren en el proceso, la metodología aplicada en el desarrollo de la clase y las normas de comportamiento y comunicación implementadas.

Por último se logró concluir que es importante que el docente asuma el rol de individuo orientador de conocimiento haciendo uso de las estrategias de comunicación, pues se lograron identificar algunos obstáculos y éxitos en el aula en relación a la interacción con la población implicada, que respectivamente se resalta la jerarquía en las clases limitando al estudiante e incluso impidiendo el desarrollo y participación activa en la clase de matemáticas lo cual recae en las estrategias de distanciamiento que según Cros ( 2003) tienen la función de mostrar la asimetría que se genera entre los docentes y los alumnos y finalmente la interacción entre docente - estudiante contribuyendo a la formación de todos los que se involucran en el proceso incentivando y apropiándose del conocimiento trabajado en grupo con ideas y procesos socializados en la clase de matemáticas que según Cros corresponde a las estrategias de aproximación.

\section{Referencias}

Cros, A., (2000), El discurso docente: entre la proximidad y la distancia. Revista Iberoamericana de Discurso y Sociedad 2 (1), Revista de Educación, Año 14, Número 26, 2008

Camacaro, Z., (2003), Convencer en clase. Argumentación y discurso docente. Barcelona: Ariel.

Rojas, F., (2009), Instrumentos discursivos para caracterizar la comunicación del profesor en el aula de matemáticas y las posibilidades de participación de los estudiantes, Universidad Autónoma de Barcelona, España.

Rojas, F. (2009). Participación en el aula de matemáticas: indicadores discursivos para caracterizar su gestión. Tesis Doctoral, Universidad Autónoma de Barcelona, Barcelona. 